\title{
Markov Random Field Model for Single Image Defogging
}

\author{
Laurent Caraffa and Jean-Philippe Tarel \\ University Paris Est, IFSTTAR, LEPSiS, \\ 14-20 Boulevard Newton, Cité Descartes, \\ F-77420 Champs-sur-Marne, France \\ Laurent.Caraffa@ifsttar.fr Jean-Philippe.Tareldifsttar.fr
}

\begin{abstract}
Fog reduces contrast and thus the visibility of vehicles and obstacles for drivers. Each year, this causes traffic accidents. Fog is caused by a high concentration of very fine water droplets in the air. When light hits these droplets, it is scattered and this results in a dense white background, called the atmospheric veil. As pointed in [1], Advanced Driver Assistance Systems (ADAS) based on the display of defogged images from a camera may help the driver by improving objects visibility in the image and thus may lead to a decrease of fatality and injury rates.

In the last few years, the problem of single image defogging has attracted attention in the image processing community. Being an ill-posed problem, several methods have been proposed. However, a few among of these methods are dedicated to the processing of road images. One of the first exception is the method in [2], [1] where a planar constraint is introduced to improve the restoration of the road area, assuming an approximately flat road.

The single image defogging problem being ill-posed, the choice of the Bayesian approach seems adequate to set this problem as an inference problem. A first Markov Random Field (MRF) approach of the problem has been proposed recently in [3]. However, this method is not dedicated to road images. In this paper, we propose a novel MRF model of the single image defogging problem which applies to all kinds of images but can also easily be refined to obtain better results on road images using the planar constraint.

A comparative study and quantitative evaluation with several state-of-the-art algorithms is presented. This evaluation demonstrates that the proposed MRF model allows to derive a new algorithm which produces better quality results, in particular in case of a noisy input image.
\end{abstract}

\section{INTRODUCTION}

Vehicle accidents may result from reduced visibility in bad weather conditions such as fog. Increasing object visibility and image contrast in foggy images grabbed from a camera inboard a vehicle thus appears to be useful for various camera-based Advanced Driver Assistance Systems (ADAS). Two kinds of ADAS can be considered. The first system consists in displaying the defogged image, for instance using a Head-Up Display. Following [1], this kind of ADAS is named Fog Vision Enhancement System (FVES). The second system combines defogging as a pre-processing with detection of vehicles, pedestrians and obstacles, in order to deliver adequate warning. An example is to warn the driver when the distance to the previous moving vehicle is too short with respect to its speed. In [4], it is shown for two types of

Thanks to the ANR (French National Research Agency) for funding, within the ICADAC project $(6866 \mathrm{C} 0210)$. detection algorithms, that defogging pre-processing allows to improve detection performances in the presence of fog.

With a linear response camera, an object of intrinsic intensity $I_{0}$ is seen with the following apparent intensity $I$ in presence of a fog with extinction coefficient $\beta$ :

$$
I=I_{0} e^{-\beta D}+\underbrace{I_{s}\left(1-e^{-\beta D}\right)}_{V}
$$

This equation is the so-called Koschmieder law, where $D$ is the object depth, and $I_{s}$ is the intensity of the sky. From (1), it can be seen that fog has two effects: first an exponential decay $e^{-\beta D}$ of the object contrast, and second an atmospheric veil $V$ is added. $V$ is an increasing function of the object depth $D$. The difficulty is that single image defogging is an ill-posed problem. Indeed, from (1), defogging requires to estimate both the intrinsic luminance $I_{0}$ and the depth $D$ at every pixel, only knowing $I$.

The first method for single image defogging is in [5], where an approximate depth-map of the scene geometry is built interactively depending of the scene. Due to the interaction, this method does not apply to camera-based ADAS. In [6], this idea of using an approximate depth-map is also investigated by proposing several simple parametric geometric models dedicated to road scenes seen in front of a vehicle. The model parameters are fit on each image by maximizing the scene depth without producing black pixels after defogging. The limit is the lack of flexibility due to simple parametric models.

The same year, a different method based on the use of color is proposed in [7]. The difficulty in the case of a road image, is that road is gray and white and thus the method does not apply correctly on this area. Then in [8], [9], [10], three defogging methods are introduced which are able to process a gray-level or as well as a color image. These three methods rely on a single principle: the use of a local spatial regularization. However, these methods are not dedicated to road images and thus the roadway area in the defogged image is usually over-contrasted. The road can be reasonably assumed to be approximately flat. In [2], it is thus proposed to introduce a planar constraint in method [10] for better defogging of the roadway area.

From 2009, several other methods for single image defogging were proposed. Nevertheless, only a few of them are able to cope with the roadway area in the image. The single image defogging problem being ill-posed, the choice of the 
Bayesian approach is adequate. A first Markov Random Field (MRF) approach was proposed in [3], based on Factorial MRF to handle the two types of fields of unknown variables: depth and intrinsic intensity. Based on our analysis of the 3D reconstruction and defogging problem in stereovision [11], we here propose to decompose the problem into two steps: first infer the atmospheric veil using a first original MRF model and second, estimate the restored image using a second original MRF model assuming that the depth-map is known. In practice, the depth-map is obtained from the map of the atmospheric veil. The proposed MRF model is generic and can easily be refined to introduce the planar constraint, which leads to better results on road images.

To compare the proposed algorithm to previously presented algorithms, we use the evaluation scheme proposed in [1] on a set of synthetic images with homogeneous fog. Algorithms are applied on foggy images and outputs are compared with the image without fog. The proposed MRF defogging method is more efficient, particularly when input image is noisy, a case which was never studied before. The article is structured as follows. Sec. II presents the MRF model for image defogging when the depth-map is assumed known. In Sec. III, the MRF model for depth inference is introduced based on the inference of the atmospheric white veil from the foggy image. The two previous models are combined in Sec. IV to derive the algorithm for MRF single image defogging. In Sec. V, a comparison is provided with five state of the art algorithms based on a quantitative evaluation on a set of 66 synthetic foggy images, illustrating the advantage of the proposed algorithm. A qualitative comparison on camera images is also proposed in Sec. VI.

\section{IMAGE DEFOGGING KNOWING THE DEPTH-MAP}

In this section, we are looking for $I_{0}$, the restored image, for input image $I$, assuming that the depth-map $D$ is known. $I_{s}$ is usually taken as the maximum intensity value over the image, see [7]. For color images, the white balance must be performed correctly before processing to achieve correct colors after defogging. The value of $\beta$, the extinction coefficient, can be obtained by fog characterization preprocessing. Examples of such pre-processing can be found for instance in [12], [13].

When the true depth-map $D$ is known, as well as $\beta$ and $I_{s}, I_{0}$ can be found in close form at each pixel, by reversing the Koschmieder law (1), see for instance [10]. The reversing of the Koschmieder law is valid only when the input image $I$ is assumed noiseless. In the presence of noise, and due to the low signal to noise ratio on remote objects, the noise on such objects is strongly emphasized after contrast restoration. To address the presence of noise, a MRF formulation is adequate and allows to better solve the single image defogging problem knowing the depth-map. The proposed MRF leads to a regularized solution, where the quality of the restored image in presence of noise is improved, even when the noise is small.

The problem of image defogging knowing the depth-map can be formulated as the maximization of the posterior prob- ability $p\left(I_{0} \mid D, I\right)$. By using the Bayes rule, this probability can be rewritten as:

$$
p\left(I_{0} \mid D, I\right) \propto p\left(I \mid D, I_{0}\right) p\left(I_{0} \mid D\right)
$$

where $p\left(I \mid D, I_{0}\right)$ is the data likelihood and $p\left(I_{0} \mid D\right)$ is the prior on the unknown $I_{0}$. In practice, the log-likelihood is minimized instead of the maximization over the probability density function. The energy derived from (2) using loglikelihood is:

$$
E\left(I_{0} \mid D, I\right)=\underbrace{E\left(I \mid D, I_{0}\right)}_{E_{\text {data } I_{0}}}+\underbrace{E\left(I_{0} \mid D\right)}_{E_{\text {prior } I_{0}}}
$$

This energy is thus the sum of two terms: the data and prior terms, which are now detailed.

\section{A. Data term}

By definition, the data term is the log-likelihood of the noise probability on the intensity, taking into account that $I_{0}$ is observed through the fog, see (1). As a consequence, it can be written as:

$$
E_{\text {data_ } I_{0}}=\sum_{(i, j) \in X} \rho\left(\frac{\left|\left(I_{0}(i, j)-I_{s}\right) e^{-\beta D(i, j)}+I_{s}-I(i, j)\right|}{\sigma}\right)
$$

where $X$ is the set of image pixels and $\rho$ is a function related to the distribution of the intensity noise with scale $\sigma$. In practice, a Gaussian distribution is usually used, i.e $\rho(t)=$ $t^{2}$.

\section{B. Prior term}

The prior term enforces the smoothness of the restored image by penalizing large differences of intensity between neighboring pixels. As a consequence, it can be written as:

$E_{\text {prior_ } I_{0}}=\lambda_{I 0} \sum_{(i, j) \in X} e^{-\beta D(i, j)} \sum_{(k, l) \in N} \rho_{g}\left(\left|I_{0}(i, j)-I_{0}(i+k, j+l)\right|\right)$

where $\lambda_{I_{0}}$ is a factor weighting the strength of the prior on $I_{0}$ w.r.t the data term, $N$ is the set of relative positions of pixel neighbors (4, 8 connectivity or other), and $\rho_{g}$ a function related to the gradient distribution of the image without fog. In practice, the identity function for $\rho_{g}$ gives satisfactory results.

Notice that an exponential decay is introduced in (5). Without this decay, and because of the exponential decay on $I_{0}$ in the data term, the effect of the data term becomes less and less important as depth increases, compared to the prior term. In such a case, the data term gets the upper hand on the prior term, and the result is over smoothed at long range distances. To avoid this effect, the exponential decay $e^{-\beta D(i, j)}$ is introduced in the prior term. Fig. 1 shows the restored image obtained with the proposed algorithm on a foggy and noisy image without the regularization due to the prior term, with the prior term without exponential decay and finally, with the prior with exponential decay. As expected, results shows that, without prior term, the noise is strongly emphasized on remote objects. Next, when the prior with exponential decay is used, the intensity of close objects 


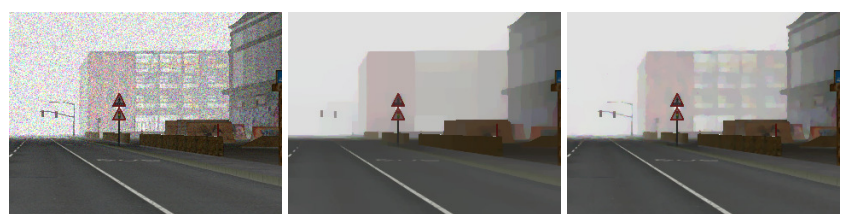

Fig. 1. Effect of the prior term $E_{\text {prior }-I_{0}}$. From left to right: without regularization i.e $\lambda_{I_{0}}=0$ a, when $\lambda_{I_{0}}=2$ and without exponential decay, when $\lambda_{I_{0}}=2$ and with exponential decay.

spreads on remote objects. With the prior with exponential decay, the smoothing effect is homogeneous with the distance and small details are kept even on remote objects.

\section{Depth versus atmospheric veil}

Rather than using the depth-map $D$ in the previous MRF model, it is equivalent to use the map of the atmospheric veil $V$. The link between the map of the atmospheric veil $V(i, j)$ and the depth-map $D(i, j)$ is:

$$
V(i, j)=I_{s}\left(1-e^{-\beta D(i, j)}\right)
$$

After substitution of $V$ in (1), the Koschmieder law is rewritten as:

$$
I=I_{0}\left(1-\frac{V}{I_{s}}\right)+V
$$

This substitution can be also performed on the data term (4):

$$
E_{\text {data_I } I_{0}}=\sum_{(i, j) \in X} \rho\left(\frac{\left|I_{0}(i, j)\left(1-\frac{V(i, j)}{I_{s}}\right)+V(i, j)-I(i, j)\right|}{\sigma}\right)
$$

and on the prior term:

$$
E_{\text {prior } I_{0}}=\lambda_{I_{0}} \sum_{(i, j) \in X}\left(1-\frac{V(i, j)}{I_{s}}\right) \sum_{(k, l) \in N} \rho_{g}\left(\left|I_{0}(i, j)-I_{0}(i+k, j+l)\right|\right)
$$

leading to a MRF model for single image defogging knowing the map of atmospheric veil $V$. One can notice, that the value of the extinction coefficient $\beta$ is not longer required in this last MRF model.

\section{ATMOSPHERIC VEIL INFERENCE}

In this section, we propose a MRF model for inferring the depth-map from a single foggy image. To avoid the sampling difficulties pointed in [3], rather than inferring the depth-map $D$, we prefer to equivalently infer the map of atmospheric veil $V$.

As previously, the MRF model for the inference of $V$ is formulated as the minimization of an energy with data and prior terms:

$$
E(V \mid I)=\underbrace{E(I \mid V)}_{E_{\text {data.V }}}+\underbrace{E(V)}_{E_{\text {prior. } V}}
$$

From (6), it is clear that the value of $V$ cannot be estimated exactly from a single image $I$, since image $I_{0}$ is also unknown. The problem is ill-posed, even when $I_{s}$ is known. We only know that the values of the atmospheric veil $V$ are subject to several constraints.

\section{A. Photometric constraint}

The first constraint is the photometric constraint, as named in [8]. It tells that $V$ is higher than zero and that $V$ is lower or equal to $I$ when the input is a gray level image, consequently:

$$
0 \leq V(i, j) \leq I(i, j)
$$

where $(i, j) \in X$. For a color image, $V$ is lower than the minimum over the Red-Green-Blue color components: $\min \left(I_{r}(i, j), I_{g}(i, j), I_{b}(i, j)\right)$.

\section{B. Planar road constraint}

For road images, the road can be assumed planar and the depth of the road plane can be used as a maximum bound for the depth-map $D$. This bound can also be written as a maximum bound on the atmospheric veil $V$, as previously in [2]. The vertical position $j_{h}$ of the line of horizon is assumed known, as well as the camera calibration with respect to the road plane. This implies that the distance to the road plane is given by:

$$
D_{\text {road }}(i, j)=\frac{\delta}{j-j_{h}} \quad \text { when } \quad j>j_{h}
$$

where $j$ is the index of a line in the image and $\delta$ is a function of the camera calibration parameters. More details are given in [1].

The maximum atmospheric veil which can be observed between the camera and the flat road is thus by substitution of (12) in (6):

$$
V_{\text {road }}(i, j)=I_{s}\left(1-e^{-\frac{\beta \delta}{\left(j-j_{h}\right)}}\right)
$$

The planar road constraint on the atmospheric veil $V$ is consequently:

$$
V(i, j) \leq V_{\text {road }}(i, j)
$$

\section{Data term}

The idea for building the data term is to maximize the value of the atmospheric veil and to respect the bounds due to the two previous constraints. By maximizing the value of $V$, we enforce that the output image will have a contrast restored as much as possible. We thus propose the following data term:

$$
E_{d a t a_{-} V}=\sum_{(i, j) \in X} E_{d a t a_{-} V}(i, j)
$$

with

$$
\left\{\begin{array}{l}
\text { if } \quad 0 \leq V(i, j) \leq \min \left(V_{\text {road }}(i, j), I(i, j)\right) \\
\quad E_{\text {data_ } V}(i, j)=\rho_{v}\left(I_{0}^{b}\left(1-\frac{V(i, j)}{I_{s}}\right)+V(i, j)-I(i, j)\right) \\
\text { else } \\
\quad E_{\text {data_ } V}(i, j)=+\infty
\end{array}\right.
$$

where $\rho_{v}$ is an arbitrary increasing function and $I_{0}^{b}$ is the initial $I_{0}$ value from which $I_{0}(i, j)$ is biased to. In practice, the identity function for $\rho_{v}$ gives satisfactory results, and allows a good propagation of the atmospheric veil in neighboring pixels. This is particularly useful when processing a graylevel input image. The choice of $I_{0}^{b}$ is important to control 

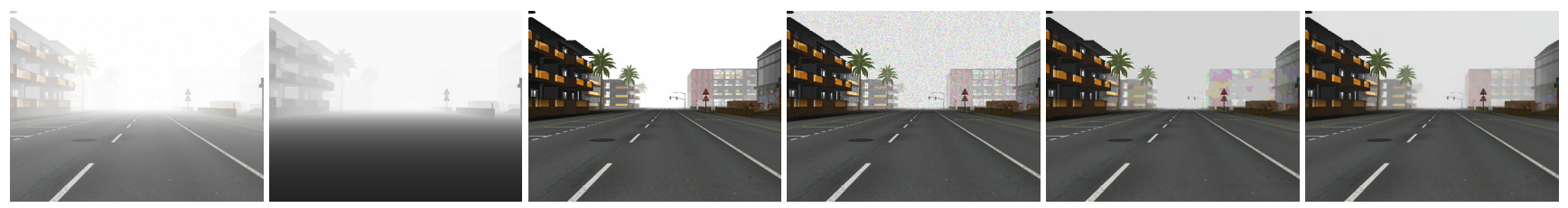

Fig. 2. Comparison of defogging results. From left ro right: the input foggy image, the atmospheric veil obtained by minimizing (10), the restoration result without regularization and without noise, the restoration result without regularization and with noise on the input image, previous method with smoothing of the input image, our restoration result.

how dark or light are the gray areas in the result image. We usually set its value as a percentage of $I_{s}$, for instance $I_{0}^{b}=0.25 I_{s}$.

\section{Prior term}

We assume that close pixels have a greater chance to have same depth, and thus the same atmospheric veil, compared to remote pixels. The prior term thus consists in a smoothness term which penalizes large jumps of the atmospheric veil in neighbors. The prior term is written as:

$$
E_{\text {prior_V }}=\lambda_{V} \sum_{(i, j) \in X} \sum_{(k, l) \in N}|V(i, j)-V(i+k, j+l)|
$$

where $\lambda_{V}$ is a factor weighting the strength of the prior on $V$ w.r.t the data term, $N$ is the set of relative positions of pixel neighbors.

When the input image is a color image, the previous MRF model is modified only with substituting $I(i, j)$ by $\min \left(I_{r}(i, j), I_{g}(i, j), I_{b}(i, j)\right)$ in (16).

\section{Single IMAGE DEFOGGING}

The proposed algorithm consists into two steps. In the first step, the atmospheric veil $V$ is obtained from $I$ by minimizing the MRF energy $E(V \mid I)=E_{\text {data_ } V}+E_{\text {prior }_{-} V}$, the sum of data and prior terms (16) and (17). When $V$ is inferred, the restored image is estimated in a second step by minimizing the energy $E\left(I_{0} \mid V, I\right)$ the sum of data and prior terms (8) and (9).

In these two steps, the two involved energies are of the form:

$$
f(Y)=\sum_{x \in X} \Phi_{x}\left(Y_{x}\right)+\sum_{x \in X, x^{\prime} \in X} \Phi_{x, x^{\prime}}\left(Y_{x}, Y_{x^{\prime}}\right)
$$

and it is known [14] that, when $\Phi_{x, x^{\prime}}$ is sub-modular, the global minimum of the problem can be obtained in polynomial time for binary variables $Y_{x}$. In our case, which is multi-label, a local minimum can be reached using the $\alpha$ expansion algorithm, which is based on the decomposition in successive binary problems.

The algorithm scheme is thus the following:

- First, compute the atmospheric veil by finding the $V$ minimizing (10) with $\alpha$-expansion optimization, using the identity function for $\rho_{v}$.

- Second, the restored image is obtained by minimizing (3) again with $\alpha$-expansion optimization, using the identity function for $\rho_{g}$. For a color input image, the last step is performed on every channel independently.

\section{RESUltS ON SYNTHETIC IMAGES}

Fig. 2 shows results for different parameters on the same synthetic input image with and without added noise. Without noise, the restored image can be computed without any regularization, i.e with $\lambda_{I_{0}}=0$. With added noise, the noise is emphasized with the distance in the output. The input image can be smoothed before restoration, but details are then lost and incorrect clusters of colors appear on remote objects. When the prior term is used during the restoration with the exponential decay, the smoothing effect is more uniform as already shown in Fig. 1. In particular, the color is more homogeneous at long range distances and small details are better restored.

TABLE I

AVERAGE ABSOLUTE ERROR BETWEEN RESTORED IMAGE AND TARGET IMAGE WITHOUT FOG, FOR 6 DEFOGGING METHODS ON 66 SYNTHETIC IMAGES WITH UNIFORM FOG. RESULTS WITHOUT NOISE ARE IN THE SECOND COLUMN, AND IN THE THIRD ONE, WITH ADDED NOISE.

\begin{tabular}{|c|c|c|}
\hline Algorithm & mean error (in gray levels) & mean error $(+$ noise std=1) \\
\hline Nothing & $81.6 \pm 12.3$ & $81.2 \pm 12.4$ \\
DCP [9] & $46.3 \pm 15.6$ & $49.2 \pm 14.4$ \\
FSS [4] & $34.9 \pm 15.1$ & \\
NBPC [10] & $50.8 \pm 11.5$ & $50.4 \pm 11.8$ \\
NBPC+PA [2] & $31.1 \pm 10.2$ & $26.4 \pm 8.7$ \\
CM+PA [15] & $19.9 \pm 11.1$ & $19.1 \pm 6.7$ \\
Proposed & $19.6 \pm 10.7$ & $16.9 \pm 5.1$ \\
\hline
\end{tabular}

We evaluate the proposed algorithm on 66 synthetic images with uniform fog from the database named FRIDA2 ${ }^{1}$. This database was introduced first in [1] and contains a ground-truth for defogging methods. The proposed algorithm is compared with five other algorithms: Dark Channel Prior (DCP) [9], Free Space Segmentation (FSS) [4], No Black Pixel Constraint (NBPC) [10], No Black Pixel Constraint and Planar road Assumption (NBPC+PA) [2], Contrast Maximization and Planar road Assumption (CM+PA) [15]. In order to experiment with noise, we present, in Tab. I, results without and with an added Gaussian noise (with standard deviation one gray level) on all the input images. With noise, the weight $\lambda_{I_{0}}$ is increased to take into account this increased noise. The value used for $I_{0}^{b}$ is $0.25 I_{s}$.

For road scenery, this table shows that the proposed algorithm is better than the five other algorithms, in particular in presence of noise. The average error is more reduced in

\footnotetext{
${ }^{1}$ www.lcpc.fr/english/products/image-databases/article/frida-foggy-roadimage-database
} 

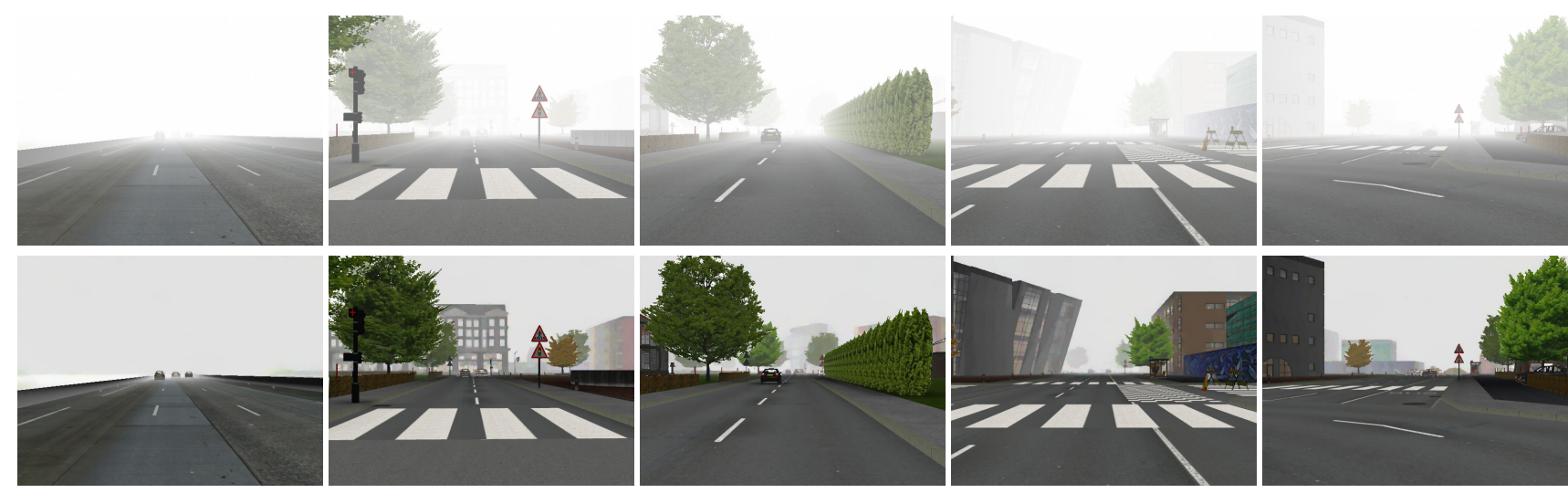

Fig. 3. Defogging results on synthetic images from FRIDA2 database. First line: the image with homogeneous fog and Gaussian noise. Second line: the obtained restored images using proposed MRF model. Notice how long range distance vehicles are better seen on the restored images.

the noisy case, in comparison to the noiseless case. This is not the case for most of the other algorithms since they are not designed to take into account input image noise. Thanks to this smoothing, the proposed algorithm is also more stable, with a standard deviation reduced to 5.1 gray levels in the noisy case, compared to 10.7 in the noiseless case. Sample results obtained on the FRIDA2 database are shown in Fig. 3. One can notice how far away vehicles are better seen in the restored images.

\section{RESULTS ON CAMERA IMAGES}

The proposed algorithm also produced nice results on camera images as illustrated on gray-level and color images in Fig. 4. The smoothing weight $\lambda_{I_{0}}$ is set to 2 for the estimation of $I_{0}$ in these experiments. One can notice that, even if the smoothing removes noise, the structures of remote objects are well preserved.

In Fig. 5, we show a comparison of the proposed algorithm with other previously proposed algorithms: [8], DCP [9], NBPC [10], NBPC+PA [2]. Only the NBPC+PA algorithm is dedicated to road images since it includes the flat road constraint, like the proposed algorithm. As a consequence, results obtained with the proposed algorithm are quite close to the one obtained using NBPC+PA algorithm. However, remote areas are better smoothed and some thin objects are better restored.

\section{CONCLUSION}

We propose a new MRF model for Bayesian defogging and derive the defogging algorithm from this model by $\alpha$-expansion optimization. The algorithm is in two steps: first, the atmospheric veil (or equivalently the depth-map) is inferred using a dedicated MRF model. In this MRF model, the flat road assumption can be introduced easily to achieve better results on road images. Once the atmospheric veil is inferred, the restored image is estimated by minimizing another MRF energy which models the image defogging in presence of noisy inputs. Evaluation on both synthetic images and real world images shows that the proposed method outperforms the state of the art in single image defogging, when an homogeneous fog is present.
In the future, we intend to investigate the case of nonhomogeneous fog. We will also study means to speed up the proposed algorithms in order to achieve close to real-time processing.

\section{REFERENCES}

[1] J.-P. Tarel, N. Hautière, L. Caraffa, A. Cord, H. Halmaoui, and D. Gruyer, "Vision enhancement in homogeneous and heterogeneous fog," IEEE Intelligent Transportation Systems Magazine, vol. 4, no. 2 , pp. 6-20, Summer 2012.

[2] J.-P. Tarel, N. Hautière, A. Cord, D. Gruyer, and H. Halmaoui, "Improved visibility of road scene images under heterogeneous fog," in Proceedings of IEEE Intelligent Vehicle Symposium (IV'2010), San Diego, California, USA, 2010, pp. 478-485.

[3] K. Nishino, L. Kratz, and S. Lombardi, "Bayesian defogging," International Journal of Computer Vision, vol. 98, pp. 263-278, 2012.

[4] N. Hautière, J.-P. Tarel, and D. Aubert, "Mitigation of visibility loss for advanced camera based driver assistances," IEEE Transactions on Intelligent Transportation Systems, vol. 11, no. 2, pp. 474-484, June 2010.

[5] S. G. Narashiman and S. K. Nayar, "Interactive deweathering of an image using physical model," in IEEE Workshop on Color and Photometric Methods in Computer Vision, Nice, France, 2003.

[6] N. Hautière, J.-P. Tarel, and D. Aubert, "Towards fog-free in-vehicle vision systems through contrast restoration," in IEEE Conference on Computer Vision and Pattern Recognition (CVPR'07), Minneapolis, Minnesota, USA, 2007, pp. 1-8.

[7] R. Tan, N. Pettersson, and L. Petersson, "Vvisibility enhancement for roads with foggy or hazy scenes," in Proceedings of the IEEE Intelligent Vehicles Symposium (IV'07), Istanbul, Turkey, 2007, pp. $19-24$.

[8] R. Tan, "Visibility in bad weather from a single image," in IEEE Conference on Computer Vision and Pattern Recognition (CVPR'08), Anchorage, Alaska, 2008, pp. 1-8.

[9] K. He, J. Sun, and X. Tang, "Single image haze removal using dark channel prior," IEEE Transactions on Pattern Analysis and Machine Intelligence, vol. 33, no. 12, pp. 2341-2353, December 2010.

[10] J.-P. Tarel and N. Hautière, "Fast visibility restoration from a single color or gray level image," in Proceedings of IEEE International Conference on Computer Vision (ICCV'09), Kyoto, Japan, 2009, pp. 2201-2208.

[11] L. Caraffa and J.-P. Tarel, "Stereo reconstruction and contrast restoration in daytime fog," in Proceedings of Asian Conference on Computer Vision (ACCV'12), Daejeon, Korea, 2012.

[12] J. Lavenant, J.-P. Tarel, and D. Aubert, "Procédé de détermination de la distance de visibilité et procédé de détermination de la présence d'un brouillard," French pattent number 0201822, INRETS/LCPC, February 2002.

[13] N. Hautière, J.-P. Tarel, J. Lavenant, and D. Aubert, "Automatic fog detection and estimation of visibility distance through use of an onboard camera," Machine Vision and Applications, vol. 17, no. 1, pp. 8-20, april 2006. 


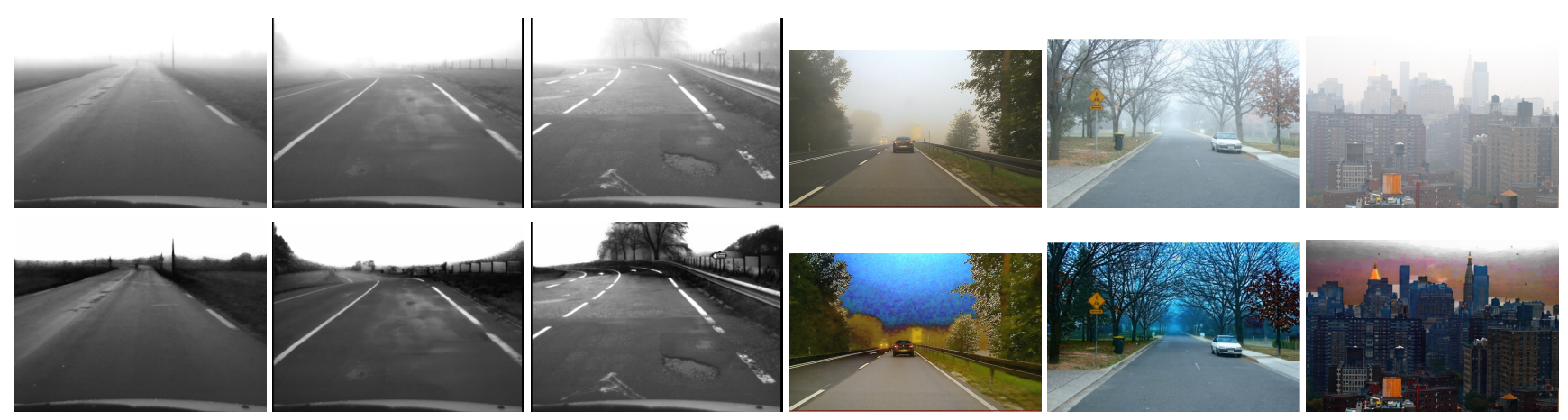

Fig. 4. Result of restoration on camera images. First line, the original image. Second line, the restored image with the proposed MRF based algorithm. The road planar constraint is used only for the 5th first images. Fourth foggy image is courtesy of Audi. Fifth foggy image is courtesy of [8]. Sixth foggy image is courtesy of [5].
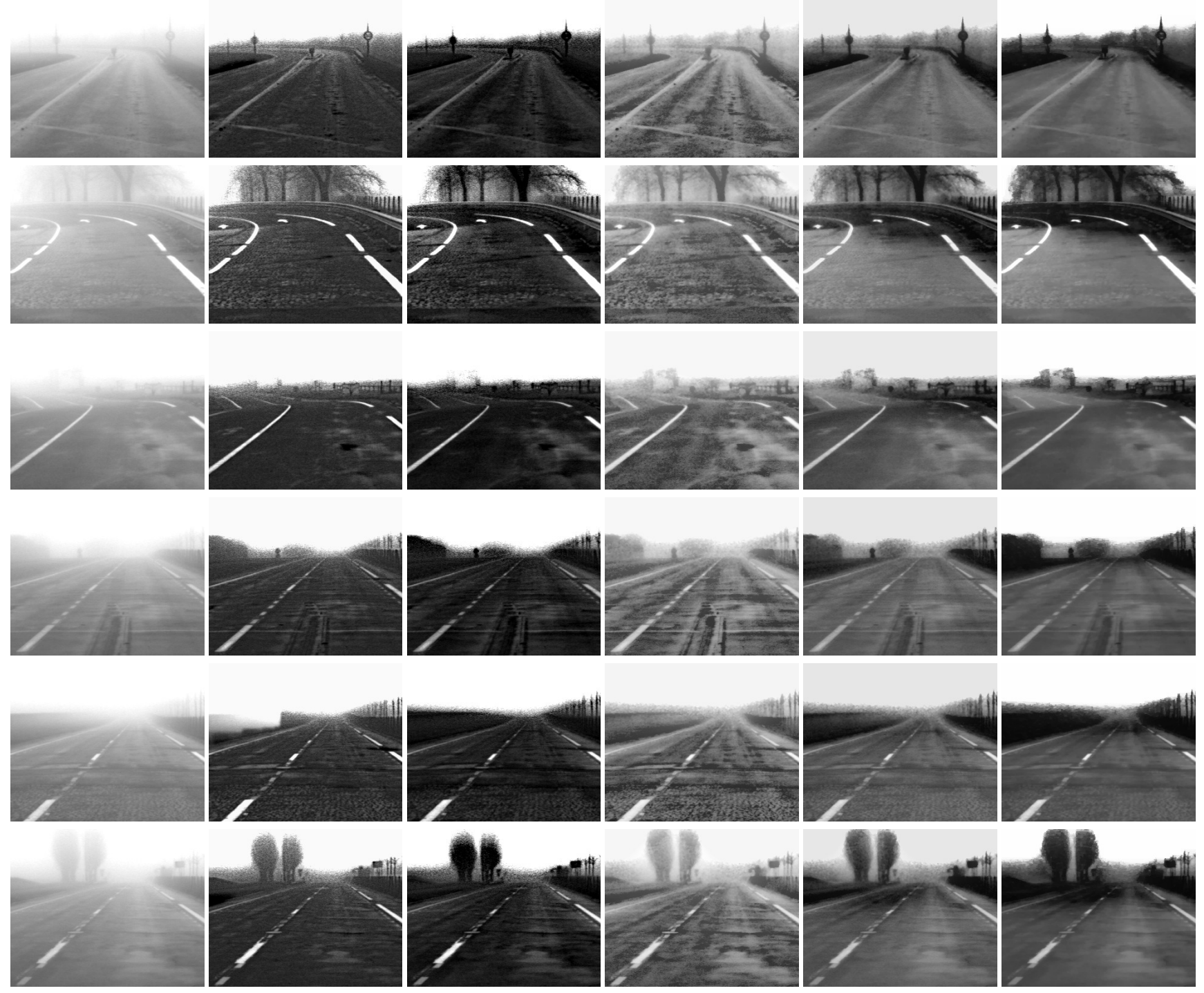

Fig. 5. Comparison on camera images. From left to right column: original foggy image, results using [8], DCP [9], NBPC [10], NBPC+PA [2], proposed MRF based algorithm.

[14] Y. Boykov, O. Veksler, and R. Zabih, "Fast approximate energy minimization via graph cuts," Pattern Analysis and Machine Intelligence, IEEE Transactions on, vol. 23, pp. 1222-1239, November 2001.

[15] H. Halmaoui, A. Cord, and N. Hautière, "Contrast restoration of road images taken in foggy weather," in Computational Methods for the Innovative Design of Electrical Devices, 2011, pp. 2057-2063. 